\title{
MARSpline model for lead seven-day maximum and minimum air temperature prediction in Chennai, India
}

\author{
$\mathrm{K}_{\text {RAMESH }}{ }^{1, *}$ and R ANITHA ${ }^{2}$ \\ ${ }^{1}$ Regional Centre, Anna University, Tirunelveli, Tamil Nadu, India. \\ ${ }^{2}$ K S Rangasamy College of Technology, Tiruchengode, Namakkal District, Tamil Nadu, India. \\ ${ }^{*}$ Corresponding author.e-mail: rameshk7n@yahoo.co.in
}

In this study, a Multivariate Adaptive Regression Spline (MARS) based lead seven days minimum and maximum surface air temperature prediction system is modelled for station Chennai, India. To emphasize the effectiveness of the proposed system, comparison is made with the models created using statistical learning technique Support Vector Machine Regression (SVMr). The analysis highlights that prediction accuracy of MARS models for minimum temperature forecast are promising for short-term forecast (lead days 1 to 3 ) with mean absolute error (MAE) less than $1^{\circ} \mathrm{C}$ and the prediction efficiency and skill degrades in medium term forecast (lead days 4 to 7 ) with slightly above $1^{\circ} \mathrm{C}$. The MAE of maximum temperature is little higher than minimum temperature forecast varying from $0.87^{\circ} \mathrm{C}$ for day-one to $1.27^{\circ} \mathrm{C}$ for lag day-seven with MARS approach. The statistical error analysis emphasizes that MARS models perform well with an average $0.2^{\circ} \mathrm{C}$ of reduction in MAE over SVMr models for all ahead seven days and provide significant guidance for the prediction of temperature event. The study also suggests that the correlation between the atmospheric parameters used as predictors and the temperature event decreases as the lag increases with both approaches.

\section{Introduction}

Timely and accurate prediction of air temperature is essential and important since it directly influences water demand, energy consumption, agricultural activity, livestock, and human livelihood and most atmospheric events such as precipitation, fog, wind speed, evapotranspiration, humidity, and pressure. Particularly, elderly people, young children, poorer communities, and outdoor workers are more vulnerable to heat stress and heat wave (Nag et al. 2009). Hot and cold waves take thousands of lives every year in India. The public, governing bodies and meteorologists need sophisticated modelling and simulation techniques for forewarning variance in surface air temperature. Accurate temperature forecasting is difficult and complex due to the several dynamic meteorological parameters involved in the event (Donald Ahrens 2011). In recent studies, statistical approaches, multiple linear regression, and support vector regression have been used in temperature prediction successfully with better accuracy (Taylor and Leslie 2005; Lin et al. 2012). Various air temperature prediction models created using statistical forecasting techniques and artificial intelligence techniques are well suited for short term (for hourly, lead one to two days) but the variance between observed and predicted is high in medium and long term predictions. The objective of this paper is to

Keywords. MARSpline; SVMr; temperature forecast. 
use non-parametric regression technique MARS to predict the lead seven days minimum and maximum surface air temperature of the densely populated location, Chennai, India and to compare its performance with the popular statistical learning technique SVMr.

The Multivariate Adaptive Regression Spline (MARS) proposed by Friedman (1991) has gained more attention in prediction due to its significant performance with a non-linear dataset. It also has good potential in modelling complex relationships between a response variable and its predictors. In addition to the above-mentioned features, MARS has excellent analytical and modelling speed on the prediction of class distributions using independent data. Recently MARS has been used in various non-linear prediction problems, data mining and knowledge discovery process. MARS has given promising results in the prediction of freshwater species distribution, finding the non-linear relationships between species and environmental variables (Leathwick et al. 2005, 2006), and prediction of energy expenditure in children (Zakeri et al. 2010). A comparative study on credit scoring reveals that MARS outperforms traditional discriminant analysis, logistic regression, neural networks, and support vector machine (SVM) approaches (Lee et al. 2006). In this study, the efficiency of MARS prediction models are compared with the models created with statistical learning method SVMr developed by Vapnik (1995). Due to its performance and computing efficiency, SVMr is being used in many real world prediction applications and in hydrological prediction applications such as, wind speed prediction (Kramer and Gieseke 2011) and prediction of daily maximum temperature using surface observed atmospheric parameters (Paniagua-Tineo et al. 2011; Ortiz-Garcia et al. 2012).

\section{Data}

The seven atmospheric parameters (listed in table 1) recorded daily in Chennai, India $\left(13^{\circ} 4^{\prime}\right.$ $\left.7.3^{\prime \prime} \mathrm{N}, \quad 80^{\circ} 14^{\prime} 48.33^{\prime \prime} \mathrm{E}\right)$ are used as predictors to forecast the next seven days minimum and maximum temperatures. The observed predictor dataset for analysis has been obtained from National Data Centre of National Centre for Environmental Prediction (NCEP), USA (http://www. ncdc.noaa.gov/oa/ncdc.html).

Based on the availability of observed data, nine years (1995-2003) of data has been used in this analysis. Data from 1996 through 2003 (8 years) are used to formulate the models and the models' performance is validated by deploying the models with one year data (1995).
Table 1. Environmental predictors used to formulate prediction models.

\begin{tabular}{lll}
\hline Sl. no. & Predictor variable & Unit \\
\hline 1 & Mean temperature & ${ }^{\circ} \mathrm{C}$ \\
2 & Mean dew point & ${ }^{\circ} \mathrm{C}$ \\
3 & Maximum sea level pressure & $\mathrm{hPa}$ \\
4 & Mean visibility & $\mathrm{km}$ \\
5 & Mean wind speed & $\mathrm{km} / \mathrm{h}$ \\
6 & Maximum wind speed & $\mathrm{km} / \mathrm{h}$ \\
7 & Precipitation & $\mathrm{mm}$ \\
$8^{\mathrm{a}}$ & Minimum temperature & ${ }^{\circ} \mathrm{C}$ \\
$9^{\mathrm{a}}$ & Maximum temperature & ${ }^{\circ} \mathrm{C}$ \\
\hline
\end{tabular}

${ }^{a}$ Daily minimum temperature is used as the eighth predictor of minimum temperature prediction models and daily maximum temperature is used as the eighth predictor of maximum temperature prediction models.

\section{Methodology}

\section{$3.1 M A R S$}

The MARS is a flexible non-parametric procedure developed by Friedman (1991) based on spline technique, an important tool for non-parametric modelling. The main advantage of MARS over the other statistical method is that it is well suited for non-linear problems like environmental parameters prediction.

The general format of MARS non-parametric regression model formulated on the dependent variable $y$ and the predictors $x$ is:

$$
y=f(x)+\varepsilon
$$

where $\varepsilon$ is the error, $f(x)$ is the unknown regression function and it is derived by:

$$
f(x)=\beta_{0}+\sum_{m=1}^{M} \beta_{m} B_{m}(x)
$$

where $\beta_{0}$ is the coefficient of the constant basis function, $B_{m}(x)$ is the $m$ th basis function, $\beta_{m}$ is the coefficient of the $m$ th basis function, and $M$ is the number of basis functions in the model.

The MARS algorithm procedure for prediction is as follows:

- Step 1. Initially finds estimation functions over different intervals and end points (knots) of each interval. Then searches for all possible knot locations for each variable.

- Step 2. Starts with the constant basis function $B_{0}(x)=1$.

- Step 3. The forward stepwise regression procedure generates all possible basis functions or the user specified number.

- Step 4. Followed by backward stepwise procedure (pruning), it removes basis functions that overfit. 
The backward pass uses GCV (generalized crossvalidation) to compare the performance of model subsets to choose the best fitting model.

$$
\operatorname{GCV}(M)=\frac{\sum_{i=1}^{N}\left[y_{i}-f_{M}\left(x_{i}\right)\right]^{2}}{N\left[1-\left(\frac{C(M)}{N}\right)\right]^{2}}
$$

where $N$ is the number of observations and $C(M)$ is the cost complexity measure of a model containing $M$ basis functions.

\section{$3.2 S V M r$}

Support vector machine regression is a statistical machine learning prediction technique capable of modelling extremely complex functions and data relationships. In SVM regression, the input
$\mathrm{X}$ is first mapped onto $m$-dimensional feature space using fixed non-linear mapping, and then a linear model is constructed in the feature space. The SVMr used in this analysis is epsilon-SVMr (ع-SVMr) (Smola and Scholkopf 2003; Farag and Mohamed 2004). The $\varepsilon-S V M r$ trains the model in the form of

$$
y(x)=f(x)+b=W^{T} \phi(x)+b
$$

for a set of training vector $C=\left\{\left(x_{i}, y_{i}\right), i=1, \ldots\right.$, $l\}$, to minimize a general risk function of the form

$$
R[f]=\frac{1}{2}\|W\|^{2}+C \sum_{i=1}^{1} L\left(y_{i}, f(x)\right)
$$

where $W$ is the linear combination of the training patterns, $\phi(x)$ is a function of projection of the

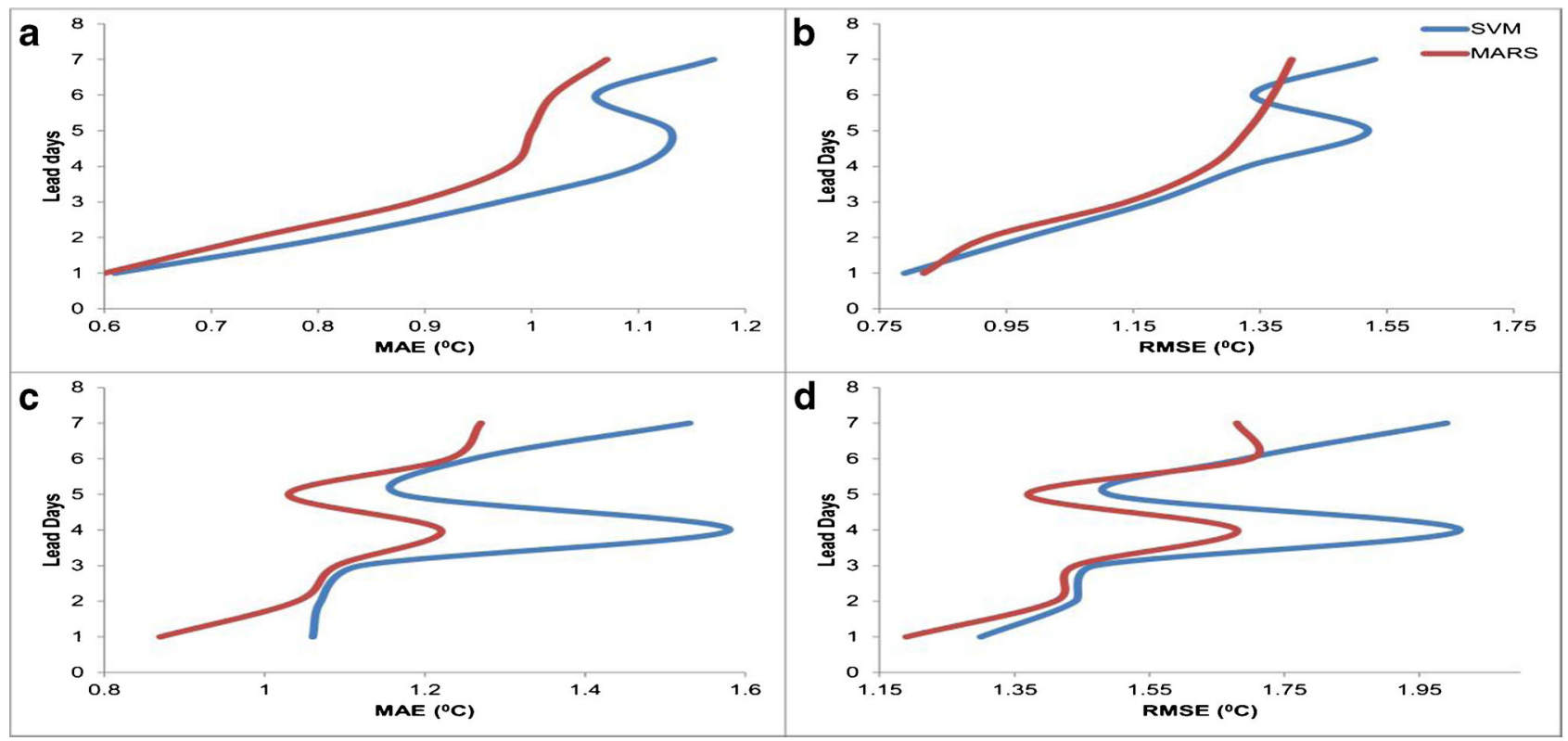

Figure 1. Temperature prediction models' performance analysis. (a) MAE minimum temperature forecast; (b) RMSE

\begin{tabular}{|c|c|c|c|c|c|c|c|c|}
\hline \multirow[b]{3}{*}{ Lead days } & \multicolumn{4}{|c|}{ Minimum temperature } & \multicolumn{4}{|c|}{ Maximum temperature } \\
\hline & \multicolumn{2}{|c|}{ MAE } & \multicolumn{2}{|c|}{ RMSE } & \multicolumn{2}{|c|}{ MAE } & \multicolumn{2}{|c|}{ RMSE } \\
\hline & $\overline{\mathrm{SVMr}}$ & MARS & $\overline{\mathrm{SVMr}}$ & MARS & $\overline{\mathrm{SVMr}}$ & $\overline{\text { MARS }}$ & $\overline{\mathrm{SVMr}}$ & $\overline{\text { MARS }}$ \\
\hline 1 & 0.61 & 0.60 & 0.79 & 0.82 & 1.06 & 0.87 & 1.30 & 1.19 \\
\hline 2 & 0.81 & 0.74 & 0.98 & 0.92 & 1.07 & 1.04 & 1.44 & 1.41 \\
\hline 3 & 0.97 & 0.89 & 1.18 & 1.14 & 1.12 & 1.09 & 1.47 & 1.44 \\
\hline 4 & 1.10 & 0.98 & 1.33 & 1.27 & 1.58 & 1.22 & 2.01 & 1.68 \\
\hline 5 & 1.13 & 1.00 & 1.52 & 1.33 & 1.17 & 1.03 & 1.49 & 1.37 \\
\hline 6 & 1.06 & 1.02 & 1.34 & 1.37 & 1.26 & 1.23 & 1.69 & 1.70 \\
\hline 7 & 1.17 & 1.07 & 1.53 & 1.40 & 1.53 & 1.27 & 1.99 & 1.68 \\
\hline
\end{tabular}
minimum temperature forecast; (c) MAE maximum temperature forecast; and (d) RMSE maximum temperature forecast.

Table 2. Performance comparison of forecast models. 
input space to the feature space, $b$ is the bias, $x_{i}$ is a feature vector of the input space with dimension $N, y_{i}$ is the output value to be estimated and $L\left(y_{i}, f(x)\right)$ is the loss function selected. In this work, the L1-loss linear SVMr function characterized by an $\varepsilon$-insensitive loss function is used.

$$
L\left(y_{i}, f(x)\right)=\left|y_{i}-f\left(x_{i}\right)\right|-\varepsilon .
$$

In order to train this model the following optimization problem is solved

$$
\min \left(\frac{1}{2}\|W\|+C \sum_{i=1}^{1}\left(\xi_{i}+\xi_{i}^{*}\right)\right)
$$

subject to the constraints

$$
\begin{array}{ll}
y_{i}-W^{T} \phi\left(X_{i}\right)-b \leq \varepsilon+\xi_{i}, & i=1, \ldots, l \\
-y_{i}+W^{T} \phi\left(X_{i}\right)+b \leq \varepsilon+\xi_{i}, & i=1, \ldots, l \\
\xi_{i}, \xi_{i}^{*} \geq 0, & i=1, \ldots, l
\end{array}
$$

The dual form of this optimization problem obtained through the minimization of the Lagterm function, constructed from the objective function and the problem constraints is

$$
\begin{aligned}
& \max \left(-\frac{1}{2} \sum_{i, j=1}^{l}\left(\alpha_{i}-\alpha_{i}^{*}\right)\left(\alpha_{j}-\alpha_{j}^{*}\right) K\left(X_{i}, X_{j}\right)\right. \\
& \left.-\varepsilon \sum_{i=1}^{l}\left(\alpha_{i}+\alpha_{i}^{*}\right)+\sum_{i=1}^{l} y_{i}\left(\alpha_{i}-\alpha_{i}^{*}\right)\right)
\end{aligned}
$$

subject to the constraints

$$
\begin{array}{r}
\sum_{i=1}^{l}\left(\alpha_{i}-\alpha_{i}^{*}\right)=0 \\
\alpha_{i}, \alpha_{i}^{*} \in[0, C]
\end{array}
$$

where $K\left(X_{i}, X_{j}\right)$ is the kernel matrix, which is formed by the evaluation of a kernel function.

The kernel function used in this study is Gaussian function as given below

$$
K\left(X_{i}, X_{j}\right)=\exp \left(-\gamma \cdot\left\|X_{i}-X_{j}\right\|^{2}\right) .
$$

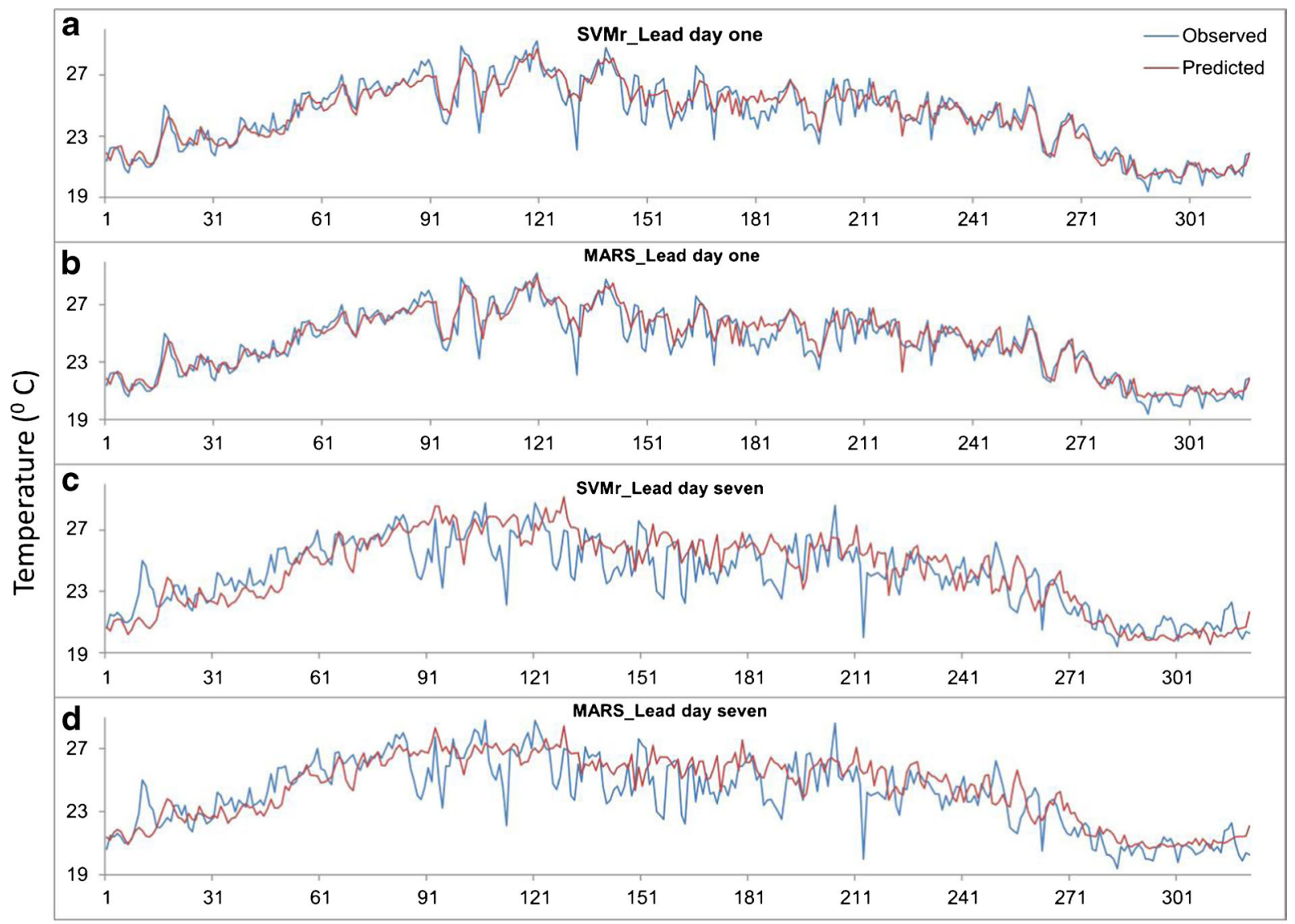

Observations (Year-1995)

Figure 2. Observed vs. predicted plot of minimum temperature prediction models lead days one and seven. 
The final form of the function $f(x)$ depends on the Lagterm multipliers $\alpha_{i}, \alpha_{j}$ as follows:

$$
f(x)=\sum_{i=1}^{l}\left(\alpha_{i}-\alpha_{i}^{*}\right) K\left(X_{i}, X\right) .
$$

\subsection{Models}

MARS minimum and maximum temperature prediction models for lead seven days were devised with the minimum threshold of 0.0005 and maximum number of basis functions 25 .

SVMr models were developed with regression SVM type 1 to predict the minimum and maximum temperatures to be felt for the next seven days. SVM has certain parameters, values of which need to be fixed appropriately for controlling undertraining and overtraining. The performance of SVM depends on the selection of the values of capacity $C, \gamma$ and loss function parameter $\varepsilon$ (Ghosh 2010). In this work, statistical data analysis tool Statistica 8 was used to create the models. The models were trained with the following SVM parameter assignments and the optimum models were preserved (model with high $R^{2}$ and least mean square error). $\mathrm{V}$-fold cross validation was done by varying capacity ' $C$ ' from 1 to 100 with interval of $1, \gamma=0.125$ and $\varepsilon$ from 0.1 to 0.5 with interval of 0.1 . Among all parameter options, the optimum results were obtained with $C=10$, $\varepsilon=0.1$ and $\gamma=0.125$. It was also noted that when $C$ value increases $(C>10)$ the error also increases and the computational time also increases.

The prediction models performance is validated by deploying the models with independent verification dataset of one year (1995). Statistical analysis, arithmetic average of the absolute error (MAE), and the square root of average squared difference (RMSE) between the forecast and observation pairs were calculated on the predicted temperature. The squaring function penalizes the temperature errors at a non-linear rate thus making larger errors more prominent. Another accuracy measure used is correlation coefficient between observed and predicted temperatures.

The absolute error or the residual $e_{i}$ is obtained by

$$
e_{i}=\left|f_{i}-y_{i}\right|
$$

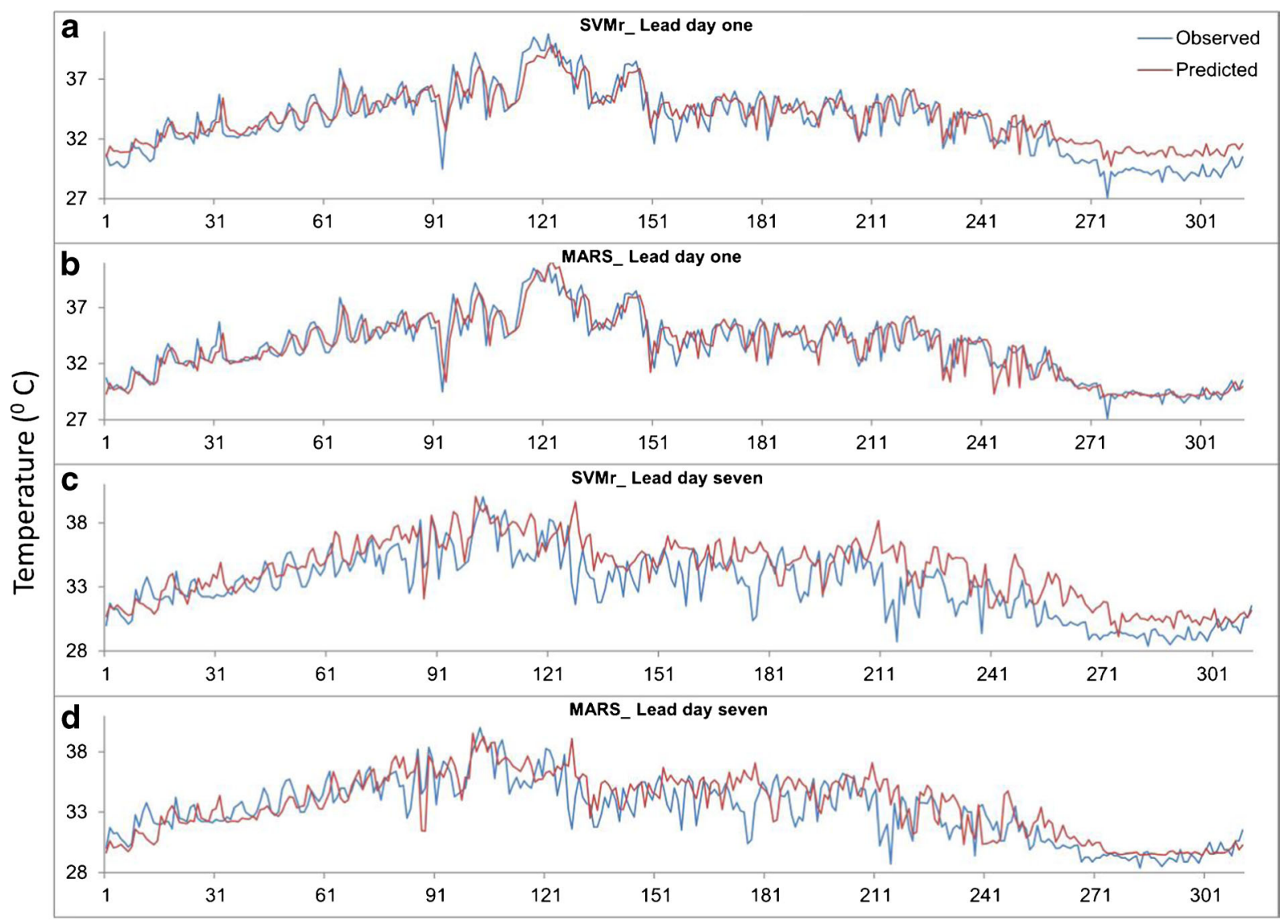

Observations (Year-1995)

Figure 3. Observed vs. predicted plot of maximum temperature prediction models lead days one and seven. 
where $f_{i}$ is the observed value and $y_{i}$ is the predicted value.

The MAE is used to measure how close forecast value is with the observed value. The MAE is given by:

$$
\mathrm{MAE}=\frac{1}{n} \sum_{i=1}^{n}\left|e_{i}\right|
$$

RMSE is the frequency used to measure the difference between the values predicted by a model and the values actually observed. It is given by:

$$
\mathrm{RMSE}=\sqrt{\frac{1}{n} \sum_{i=1}^{n} e_{i}^{2}} .
$$

\section{Result and discussion}

Statistical error analysis has been done on the forecast output generated for validating the performance accuracy of the models.

\subsection{Minimum temperature forecast model assessment}

The summary of the statistical comparison (MAE and RMSE) of SVMr and MARS minimum temperature forecast models is illustrated in figure 1 and table 2. The analysis specifies that MARS models for minimum temperature prediction are significantly good for prediction when compared with SVMr models (MAE is less than $1^{\circ} \mathrm{C}$ up to lead day five and less than $1.1^{\circ} \mathrm{C}$ for day six and seven for MARS models). The RMSE analysis also highlights that both models are prominent for short period of prediction (RMSE less than $1^{\circ} \mathrm{C}$ for day one and two). The correlation coefficient between observed and predicted values for day-one determines that the predicted values are $93 \%$ correlated with the observed. It is also renowned that correlation between observed and predicted values is above $80 \%$ for all seven lead days with MARS models. The maximum absolute error for minimum temperature warning is $3.4^{\circ} \mathrm{C}$ for lead day one and $5.4^{\circ} \mathrm{C}$ for lead day seven.

The observed versus predicted plot for both models for all lead days shows cold bias for the

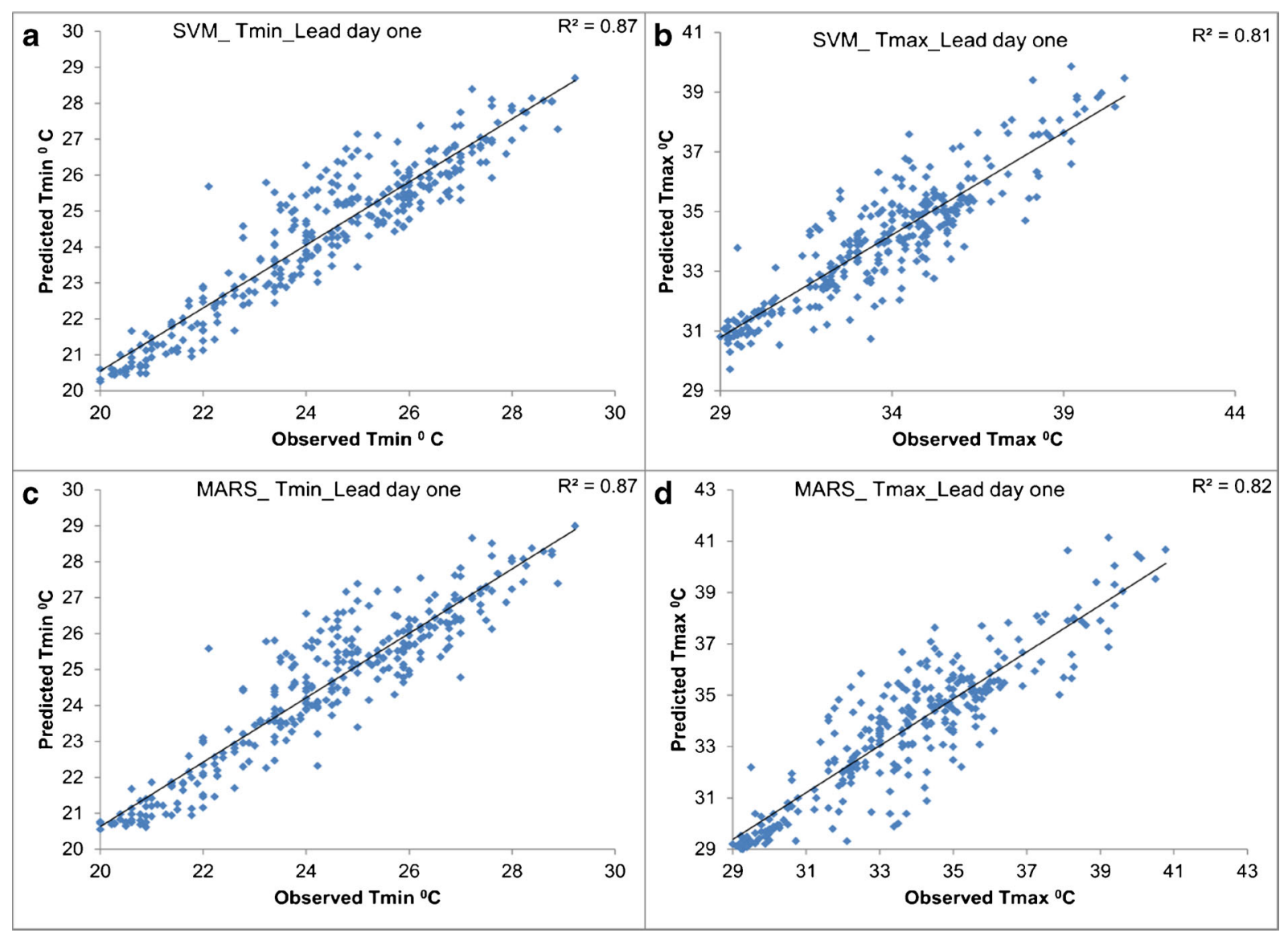

Figure 4. Plot of forecast with observed temperature for lead day one. 
months January to March and shows warm bias in the remaining days of the year (figure 2). The correlation coefficient analysis on observed and predicted values, and $R^{2}$ for models created with both approaches are relatively same for both models and it does not help to converge to a conclusion. The error analysis emphasizes that MARS models are significantly better than SVMr models in prediction accuracy.

\subsection{Maximum temperature forecast model assessment}

Figure 3 shows the observed versus predicted temperature plot for lead days one and seven for SVMr and MARS for maximum temperature estimation. The analysis on maximum temperature prediction models' outcome indicates that MARS model has coherence throughout the season, whereas SVMr shows warm bias. The comparative study on the forecast by MARS models and SVMr models states that MAE of MARS models are $0.2^{\circ} \mathrm{C}$ less for lead day one, four, five and seven when compared with SVMr models. Among the models formulated, the models for lesser lead days produce better accuracy with least MAE, RMSE and higher correlation between observed and predicted temperature. As in minimum temperature prediction, the performance degrades on higher lead days for maximum temperature prediction.

When compared with similar work with nearly same meteorological variables (Paniagua-Tineo et al. 2011), the MARS-based prediction models give better prediction with $1.19^{\circ} \mathrm{C}$ of RMSE for $24 \mathrm{~h}$ maximum temperature prediction. This approach also highlights that the atmospheric parameter used in the analysis has better correlation in predicting when compared with the minimum and maximum temperature estimation done with the MODerate-resolution Imaging Spectroradiometer (MODIS) data in east Africa (Lin et al. 2012).

The analysis also suggests that the forecast accuracy is higher for minimum temperature when compared with maximum temperature prediction (figure 4). The other advantage of this model is that once the model is created, it is possible to forecast the next seven days' temperature with the daily eight atmospheric parameters observed in the study area with less computation.

\section{Conclusion}

In this paper, a Multivariate Adaptive Regression Spline approach has been presented for forecasting lead seven days' minimum and maximum surface air temperature in Chennai. The prediction accuracy obtained is compared with Support Vector Machine regression models. Based upon the results obtained in prediction analysis and in consequent comparative analysis, it has been found that the models employing the MARS technique have consistently outperformed the models created using SVMr. This study also suggests that the forecast accuracy is higher for minimum temperature when compared with maximum temperature prediction. Prediction analysis also accentuates that the coherence between the temperature and the atmospheric parameters selected for analysis decreases as the lead increases. MARS methodology employed in this study has significant benefits and it could be implemented in real-time operations as an aid to forecasting.

\section{References}

Donald Ahrens C 2011 Essentials of meteorology - An invitation to the atmosphere; VI edn, Cengage Learning.

Farag Aly and Mohamed Refaat M 2004 Regression using Support Vector Machines: Basic Foundations; Tech. Rep. CVIP Laboratory, University of Louisville.

Friedman Jerome H 1991 Multivariate adaptive regression spline; Ann. Stat. 19 1-141.

Ghosh S 2010 SVM-PGSL coupled approach for statistical downscaling to predict rainfall from G C M output; J. Geophys. Res. 115 D22102, doi:10.1029/2009JD013548.

Kramer Oliver and Gieseke Fabian 2011 Short-term wind energy forecasting using support vector regression, soft computing models in industrial and environmental applications; 6th Int. Conf. SOCO 2011; Adv. Intelligent Soft Comput. 87 271-280.

Leathwick J R, Rowe D, Richardson J, Elith J and Hastie T 2005 Using multivariate adaptive regression splines to predict the distributions of New Zealand's freshwater diadromous fish; Freshw. Biol. 50 2034-2052.

Leathwick J R, Elith J and Hastie T 2006 Comparative performance of generalized additive models and multivariate adaptive regression splines for statistical modelling of species distributions; Ecol. Model. 199 $188-196$.

Lee Tian-Shyug, Chiu Chih-Chou, Chou Yu-Chao and Lu Chi-Jie 2006 Mining the customer credit using classification and regression tree and multivariate adaptive regression splines; Comput. Stat. Data Anal. 50 1113-1130.

Lin Shengpan et al. 2012 Evaluation of estimating daily maximum and minimum air temperature with MODIS data in east Africa; Int. J. Appl. Earth Obs. 18 $128-140$.

Nag P K and Nag A 2009 Vulnerability to heat stress: Scenario in western India; National Institute of Occupational Health, Ahmedabad.

Ortiz-Garcia E G et al. 2012 Accurate local very shortterm temperature prediction based on synoptic situation support vector regression banks; Atmos. Res. 107 $1-8$.

Paniagua-Tineo et al. 2011 Prediction of daily maximum temperature using a support vector regression algorithm; Renew. Energy 36 3054-3060. 
Smola Alex J and Scholkopf Bernhard 2003 A Tutorial on support vector regression; Statistics and Computing 14 199-222.

Taylor Andrew A and Leslie Lance M 2005 A single-station approach to model output statistics temperature forecast error assessment; Wea. Forecasting 20 1006-1019.
Vapnik V 1995 The Nature of Statistical Learning Theory; Springer, New York.

Zakeri I F, Adolph A L, Puyau M R, Vohra F A and Butte N F 2010 Multivariate adaptive regression splines models for the prediction of energy expenditure in children and adolescents; J. Appl. Physiol. 108 128-136.

MS received 20 September 2013; revised 13 December 2013; accepted 31 December 2013 